\title{
A novel system for the classification of diseased retinal ganglion cells
}

\author{
JAMES R. TRIBBLE, ${ }^{1}$ STEPHEN D. CROSS,${ }^{1}$ PAULINA A. SAMSEL,${ }^{1}$ FRANK SENGPIEL, ${ }^{2}$ AND \\ JAMES E. MORGAN ${ }^{1,3}$ \\ ${ }^{1}$ Optometry and Vision Sciences, Cardiff University, Cardiff, Wales, UK \\ ${ }^{2}$ Cardiff School of Biosciences, Cardiff University, Cardiff, Wales, UK \\ ${ }^{3}$ School of Medicine, Cardiff University, Heath Park, Cardiff, Wales, UK
}

(ReCEIVEd January 30, 2014; ACCEPTED July 31, 2014)

\begin{abstract}
Retinal ganglion cell (RGC) dendritic atrophy is an early feature of many forms of retinal degeneration, providing a challenge to RGC classification. The characterization of these changes is complicated by the possibility that selective labeling of any particular class can confound the estimation of dendritic remodeling. To address this issue we have developed a novel, robust, and quantitative RGC classification based on proximal dendritic features which are resistant to early degeneration. RGCs were labeled through the ballistic delivery of DiO and DiI coated tungsten particles to whole retinal explants of 20 adult Brown Norway rats. RGCs were grouped according to the Sun classification system. A comprehensive set of primary and secondary dendrite features were quantified and a new classification model derived using principal component (PCA) and discriminant analyses, to estimate the likelihood that a cell belonged to any given class. One-hundred and thirty one imaged RGCs were analyzed; according to the Sun classification, 24\% $(n=31)$ were $\mathrm{RGC}_{\mathrm{A}}, 29 \%(n=38) \mathrm{RGC}_{\mathrm{B}}, 32 \%(n=42) \mathrm{RGC}_{\mathrm{C}}$, and $15 \%(n=20) \mathrm{RGC}_{\mathrm{D}}$. PCA gave a 3 component solution, separating RGCs based on descriptors of soma size and primary dendrite thickness, proximal dendritic field size and dendritic tree asymmetry. The new variables correctly classified 73.3\% $(n=74)$ of RGCs from a training sample and $63.3 \%(n=19)$ from a hold out sample indicating an effective model. Soma and proximal dendritic tree morphological features provide a useful surrogate measurement for the classification of RGCs in disease. While a definitive classification is not possible in every case, the technique provides a useful safeguard against sample bias where the normal criteria for cell classification may not be reliable.
\end{abstract}

Keywords: Retinal ganglion cells, Classification, Rat, Retinal degeneration

\section{Introduction}

Inner retinal degeneration, in particular of the retinal ganglion cells (RGCs), is a hallmark of glaucoma (Qu et al., 2010) and of interest in many retinal diseases (Barber et al., 1998; Votruba et al., 1998; Williams et al., 2010). The study of RGC morphological and functional changes is vital in understanding disease progression and visual outcomes. While the primate provides the closest match to the human eye, the rat and mouse offer more tractable models for studying retinal degeneration.

Electrophysiological and morphological studies of single RGCs have revealed the functional and structural basis for the classification of RGCs. The matching of Boycott and Wassle's (1974) morphologically described cat $\alpha$ and $\beta$ cells to Enroth-Cugell and Robson's (1966) functionally described cat $Y$ and X cells highlighted the close relationship between structure and function in RGCs. The intrinsic electrical properties of the cat RGC are robust indicators

Address correspondence to: James E. Morgan, School of Optometry and Vision Sciences, Cardiff University, Maindy Road, Cardiff CF24 4LU, Wales, UK. E-mail: morganje3@cardiff.ac.uk of RGC type (O'Brien et al., 2002). RGC receptive field sensitivity maps to the dendritic field as a function of bipolar cell connectivity (Brown et al., 2000).

The shape and branching of the dendritic tree have been used to define the subclassification of RGCs in healthy adult and developing retina. The morphological classification of RGC types in the cat (Hochstein \& Shapley, 1976; Citron et al., 1988; Coelho et al., 2002), primate (Peichl, 1991; Dacey, 1993), mouse (Sun et al., 2002b; Coombs et al., 2006), and rat (Huxlin \& Goodchild, 1997; Sun et al., 2002a) is comprehensive, with many cells matching functionally described types.

By contrast, RGC classification in retinal degenerative diseases where the dendritic architecture is altered remains problematic. Recent studies have reported reductions in dendritic field size and branching density in diseased RGCs (Weber et al., 1998; Shou et al., 2003; Leung et al., 2011; Kalesnykas et al., 2012; Feng et al., 2013). The classification of RGCs by these same measurements (dendritic field diameter, branching density, and stratification) may be unreliable in disease states, particularly in rodent eyes where the features defining RGC classes can be subtle. Without classification, bias in the labeling of any given 
RGC type could show artifactual shrinkage or recovery of the dendritic tree following treatment.

In this study we explore the possibility that a robust RGC classification system can be based on dendrite features that are relatively resistant to the effects of early disease. We base this on the observation that early to moderate RGC damage consistently affects tertiary dendrites, leaving more proximal dendritic structures intact (Weber et al., 1998; Morgan et al., 2006; Williams et al., 2010). This concept is illustrated in Fig. 1 based on modeling of typical Sholl profiles in normal and diseased tissue (Weber et al., 1998; Morgan et al., 2006; Williams et al., 2010).

Our hypothesis is that a new set of parameters based on primary and secondary dendrite features can be used to indicate the class composition of a population of labeled RGCs from diseased retinas and act as a check against the selective labeling of cells from any given class. We therefore determined the extent to which structural features of primary and secondary dendrites could be used to separate cell types and derive a new classification system.

\section{Materials and methods}

Experiment procedures were conducted in accordance with the guidelines of the Society for Neuroscience and Home Office (UK) regulations governing the use of animals in research.

\section{DiOlistic labeling}

Twenty retired breeder Brown Norway rats, aged 4-6 months, were killed by overdose of $\mathrm{CO}_{2}$. Death was confirmed by cervical dislocation and the eyes immediately enucleated. The retinas were dissected in Hank's balanced salt solution (Life Technologies) and orientated using a cut in the nasal quadrant. Dissected retinas were flat mounted on cell culture inserts (Millipore $0.4 \mu \mathrm{m}$ pore, Fisher Scientific) and DiOlistically labeled following a modified protocol of Gan (Gan et al., 2000). A Helios gene gun (BioRad) was used to fire $1.7 \mu \mathrm{m}$ tungsten particles coated with 1,1'-dioctadecyl-3,3,3',3'-tetramethylindocarbocyanine perchlorate

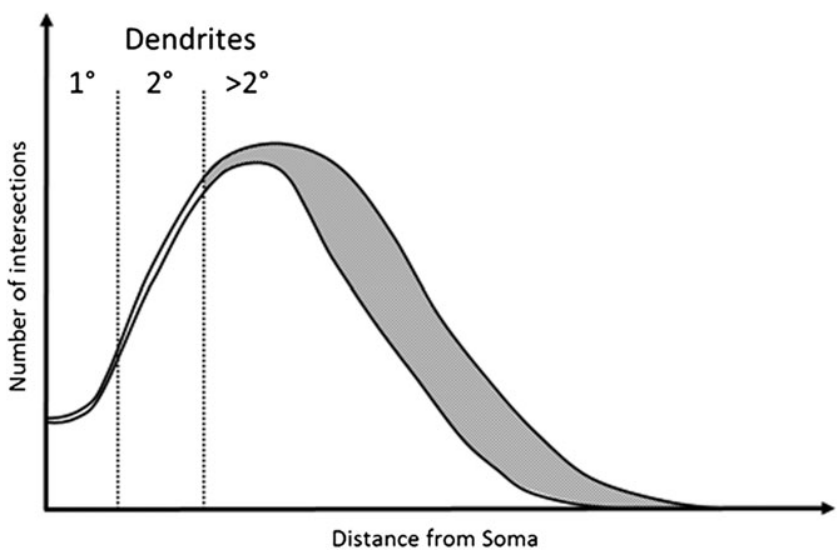

Fig. 1. Example of a degenerated Sholl plot. Graphical representation of a Sholl plot from healthy and degenerate RGCs as observed in Williams et al. (2010), Weber et al. (1998), and Morgan et al. (2006). Under moderate degeneration, Sholl plots show high deviation (gray shaded area) from healthy plots distal to the soma, with little deviation proximal to the soma. Primary and secondary dendrites occur within this proximal region and are therefore more likely preserved. Measurements of primary and secondary dendrites may therefore provide robust and stable classification criteria.
(DiI) and 3,3'-dioctadecyloxacarbocyanine perchlorate (DiO) (both Life Technologies) into the retina at $689.5 \mathrm{kPa}$ (100 psi) and a gun nozzle to retinal surface distance of $4 \mathrm{~cm}$. Aggregated particles were excluded by placing a cell culture insert (BD Falcon $3.0 \mu \mathrm{m}$, BD Biosciences) between the retina and gene gun. Retinas were then cultured in Neurobasal-A medium for $30 \mathrm{~min}$ at $37^{\circ} \mathrm{C}$. Retinas were flat mounted ganglion layer up on Histobond ${ }^{\circledR}$ coated slides (Fisher Scientific), fixed with $4 \%$ paraformaldehyde ( $\mathrm{pH} 7.4$ ) for $20 \mathrm{~min}$, and washed with phosphate buffered saline (PBS). TO-PRO-3 iodide (Life Technologies) diluted 1:1000 in PBS was applied for visualization of ganglion cell and inner nuclear layers in order to measure stratification of RGCs in the inner plexiform layer (IPL). Retinas were then mounted in ProLong ${ }^{\circledR}$ gold antifade reagent (Life Technologies). A Zeiss LSM 510 confocal microscope (Carl Zeiss AG, Germany) with a x20 air objective (NA 0.8) was used to capture multichannel z-stack images $(1024 \times 1024$ pixels, $0.54 \mu \mathrm{m} /$ pixel, slice thickness $1 \mu \mathrm{m}$ ) of single DiI and DiO labeled RCCs, with TO-PRO-3 stained ganglion cell to inner nuclear layers. RGC distance from the optic nerve in the $x$ and $y$ plane was recorded using a custom scanning stage attached to the microscope.

\section{Analysis of new RGC parameters}

Only those RGCs with an axon traceable toward the optic nerve and a dendritic tree easily distinguishable from those of other cells were included. In addition, RGCs whose primary dendrite origin at the soma was obscured was not included. In the analysis, the following dendrite parameters were determined:

- The number of primary dendrites (PD) - i.e., those originating directly from the soma.

- The distance from the soma to the primary branch point was measured for each primary dendrite using the simple neurite tracer plugin (Longair et al., 2011) in Fiji (Schindelin et al., 2012) and a mean primary dendrite length (PDL) given for each cell.

- Primary branch point field (PBPF) area: In Z-collapsed images, the primary branch points were connected and the area of the resultant field measured to give the $\mathrm{PBPF}$ area.

- PBPF center of gravity offset (CGO) from the soma: As a measure of asymmetry in the dendritic tree, the PBPF's center of gravity offset from the soma was measured. The PBPF centroid was found and its distance from the soma center measured. This was expressed as a percentage of the diameter of the PBPF to account for RGC size variation.

- Secondary branch point field (SBPF) area.

- SBPF center of gravity offset (CGO) from the soma.

- PD asymmetry: As a further measure of asymmetry, the distribution of PD about $360^{\circ}$ of the cell soma was measured using the oval profile plugin in Image J (NIH, US). In Z-collapsed images, a circle with a radius of $25 \mu \mathrm{m}$ from the soma center was drawn, and the angle at which each primary dendrite intersected the circle measured. The angle formed between the primary dendrite immediately clockwise and counter clockwise of the axon was taken so that a larger angle would represent greater asymmetry in the PBPF.

- Proximal branching density: As a measure of branching density in the proximal dendritic tree, the PBPF area was expressed as a percentage of the SBPF area.

- Soma diameter: RGC soma diameter was also measured by drawing a polygon around the soma in Z-collapsed images, the resulting area was converted to a circle and the diameter calculated. 
- Distance of RGC soma in the $X$ and $Y$ plane from the optic nerve head $(\mathrm{ONH})$ center, also expressed as a vector length.

- PD cross-sectional area: As a measure of primary dendrite thickness at the origin from the soma, primary dendrite traces derived from the simple neurite tracer plugin were resliced to give a cross-sectional view and the area at the origin measured.

- PD Feret diameter: As a further measure of dendrite thickness, the minimum and maximum Feret diameters of the resliced dendrite were measured.

\section{Comparison to existing classification}

RGCs were classified according to Sun (Sun et al., 2002a) on the basis of dendritic field diameter, dendrite stratification within the IPL, soma diameter, and branching density. Sholl analysis using FastSholl plug-in (Gutierrez \& Davies, 2007) for MATLAB (MathWorks) was conducted on all cells. Cells were grouped according to Sun (Sun et al., 2002a) and the mean and SD of the new parameters were calculated for each resulting group. A principal component analysis (PCA) was then performed in SPSS (version 18.0, IBM) to reveal factors driving separation and a discriminant analysis (SPSS) performed in order to reveal if the new parameters could classify into the same groupings as Sun. RGCs were randomly assigned to either a training data sample or hold-out sample. Each RGC was assigned a random number from a chi-square distribution with 1 degree of freedom using the random number generator function in SPSS. RGCs were then sorted by size of random number in ascending order with the first 30 RGCs assigned to a hold-out sample and the remaining 101 RGCs to the training sample.

\section{Results}

\section{$R G C$ imaging and Sun classification}

One-hundred and thirty one RGCs from 20 retinas imaged were included in the analysis based on the described exclusion criteria. The retinal locations of 114 RGCs are shown in Fig. 2 (the locations of 26 cells were not measured). The mean Sholl profile for each RGC type is plotted in Fig. 3. Classification according to the criteria of Sun et al. (2002a) gave comparable percentages of cell types in the overall population, reported here as present study percentage (Sun percentage), at $24 \%(18 \%) \mathrm{RGC}_{\mathrm{A}}, 29 \%(30 \%) \mathrm{RGC}_{\mathrm{B}}, 32 \%$ (36\%) $\mathrm{RGC}_{\mathrm{C}}$, and $15 \%$ (18\%) $\mathrm{RGC}_{\mathrm{D}}$. Typical cells for each of the 4 classes are shown in Fig. 4. Our sample therefore provides a similar breakdown of the RGC population for the derivation of classification parameters. The mean and standard deviation according to Sun type for all classification parameters measured are detailed in Table 1.

\section{Retinal eccentricity does not affect classification}

One-way ANOVA with post-hoc Bonferroni showed no significant difference between RGC types with respect to retinal eccentricity when expressed as vector length $(P>0.05)$. This confirmed that labeling was not regionally biased. RGC distance from the $\mathrm{ONH}$ center expressed as distance in $x, y$ and vector distance all showed no correlation to any variables $(P>0.05)$ demonstrating that retinal eccentricity had no effect on variable measurements. The classification of RGCs into types according to Sun was also unaffected by retinal eccentricity as dendritic field diameter and soma diameter showed no correlation to vector length ( $P>0.05$, Pearson's correlation).

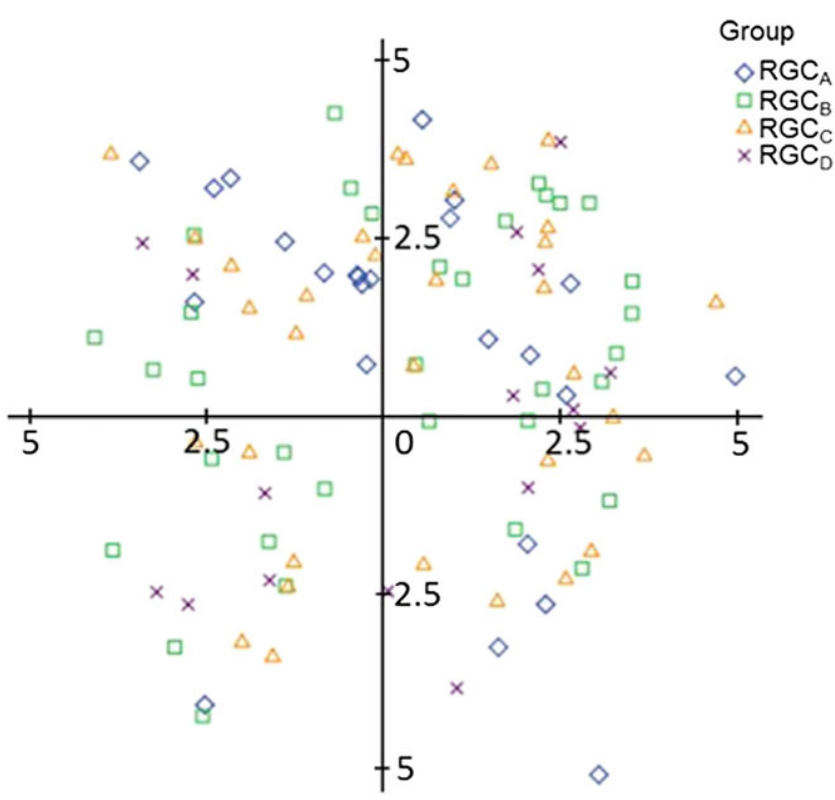

Fig. 2. Distribution of recorded RGCs. Retinal eccentricity of 114 RGCs shown as distance from the ONH. RGC types show an even spread through retinal quadrants and central/peripheral retina demonstrating minimal regional labeling biases in DiOlistic delivery. Axes show distance from $\mathrm{ONH}$.

\section{RGCs differ in size and asymmetry of the proximal dendritic tree}

A PCA was performed in order to determine the variables that contribute to the variation in morphology between RGC types and are thus the most useful in discriminating RGC types. PCA loses power and relevance if variables included are not correlated. RGC distance from the $\mathrm{ONH}$ in the $x$ and $y$ plane and as vector length were all discarded as variables due to the lack of correlation (as discussed above). Pearson's correlation and Spearman's rank tests were performed on normally and non-normally distributed variables, respectively. PDL and proximal branching density showed a significant correlation with fewer than half of the total variables $(P>0.05)$ and so were excluded from PCA. The remaining variables all showed a significant correlation $(P<0.05)$ to at least $3 / 4$ of total variables and so were included.

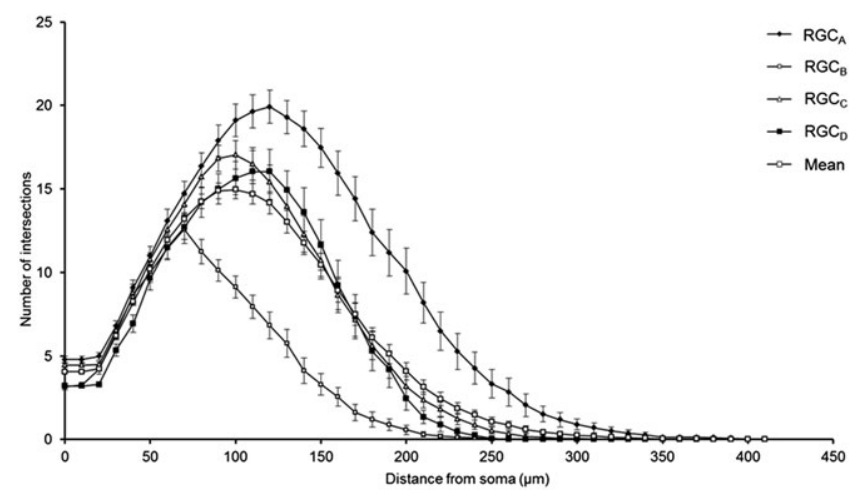

Fig. 3. Variation of Sholl plot between RGC types. Mean Sholl plot $(n=131)$ and Sholl plots of RGCs grouped according to type reveal the variation within the pooled population. The plots highlight how analysis of an unclassified population of RGCs could suffer from intrinsic bias through disproportionate numbers of cell types; the leftward shift observed in degeneration is also seen when comparing healthy types. Classification is therefore critical in removing type specific biases. Error bars show SEM. 

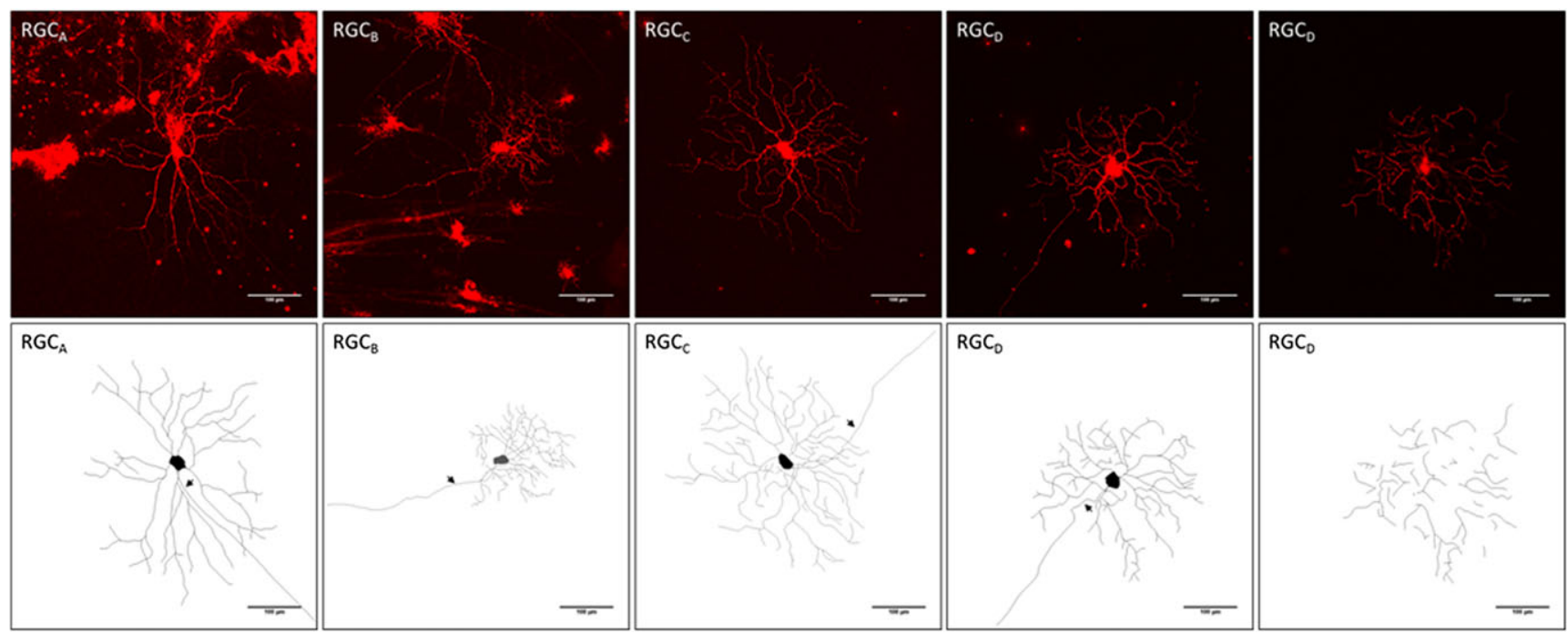

Fig. 4. RGC types. RGC types display a characteristic morphology, represented by z-compressed confocal images and tracings of typical cells of each type (Bistratified $\mathrm{RGC}_{\mathrm{D}}$ is depicted as inner and outer IPL stratifications, left and right tracings, respectively). Arrows denote axon. Scale bar $=100 \mu \mathrm{m}$.

A PCA with extraction of eigenvalues $>1$ through an oblique (promax) rotation with Kaiser normalization was performed on the training sample. A Kayser Meyer Olkin measure of sampling adequacy (0.844) indicated a sufficiently large sample size while Bartlett's test of sphericity $(P<0.0001)$ allowed rejection of the null hypothesis that the correlation matrix was an identity matrix.

PCA produced a 3 component solution that accounted for $77.1 \%$ of the variance within the data. The rotated pattern matrix showing the variable contributions to the components is shown in Table 2. The first component comprised soma diameter, primary dendrite cross-sectional area, and minimum and maximum Feret and accounted for $45.5 \%$ of the variance in the data. This component represents the size of the RGC soma and primary dendrite thickness. The second component comprised the number of PD and PBPF and SBPF areas representing proximal dendritic tree size (accounting for $21.2 \%$ of the variance). The third component comprised both PBPF and SBPF CGO and PD range (accounting for $10.4 \%$ of the variance) thus representing asymmetry in the proximal dendritic tree. The contribution of the individual variables to the 3 dimensional separation of the RGC population is shown in Fig. 5.

\section{Proximal dendritic measurements can define RGC types}

RGCs in the training sample $(n=101)$ were grouped using discriminant analysis. RGCs were grouped according to Sun et al. (2002a). Prior probability for each RGC group was calculated based on group percentages of the training sample population to account for frequency of RGC types among a population. Discriminant analysis generated 3 discriminant functions for the separation of RGCs into groups. A chi-square statistical test of the discriminant functions showed that function 3 had no discriminating ability $(P>0.05)$ and so does not contribute to the separation of RGC groups. The loadings of the individual variables onto the discriminant functions are shown in Table 3 . The first discriminant function accounted for $72 \%$ of between group

Table 1. RGC morphology measurements

\begin{tabular}{|c|c|c|c|c|}
\hline Measurement & $\mathrm{RGC}_{\mathrm{A}}$ & $\mathrm{RGC}_{\mathrm{B}}$ & $\mathrm{RGC}_{\mathrm{C}}$ & $\mathrm{RGC}_{\mathrm{D}}$ \\
\hline Soma diameter $(\mu \mathrm{m})$ & $22.7 \pm 3.5$ & $15.6 \pm 2.3$ & $18.8 \pm 3.0$ & $18.7 \pm 2.5$ \\
\hline $\operatorname{PDL}(\mu \mathrm{m})$ & $25.8 \pm 13.6$ & $22.5 \pm 14.8$ & $22.2 \pm 9.0$ & $20.1 \pm 8.2$ \\
\hline PDN & $4.3 \pm 1.1$ & $3.2 \pm 0.8$ & $3.9 \pm 0.8$ & $3.1 \pm 0.7$ \\
\hline PBPF area $\left(\mu \mathrm{m}^{2}\right)$ & $1664 \pm 1081$ & $758 \pm 791$ & $1716 \pm 1052$ & $724 \pm 486$ \\
\hline $\mathrm{SBPF}$ area $\left(\mu \mathrm{m}^{2}\right)$ & $12,352 \pm 8383$ & $3391 \pm 3291$ & $7830 \pm 5893$ & $3981 \pm 2184$ \\
\hline COG PBPF $(\%)$ & $22.9 \pm 12.3$ & $56.8 \pm 55.3$ & $23.4 \pm 28.3$ & $68.3 \pm 43.0$ \\
\hline COG SBPF (\%) & $17.6 \pm 7.1$ & $37.6 \pm 35.7$ & $19.4 \pm 9.7$ & $32.2 \pm 18.8$ \\
\hline PD asymmetry $\left({ }^{\circ}\right)$ & $78.8 \pm 38.6$ & $166.0 \pm 74.8$ & $111.4 \pm 62.4$ & $182.5 \pm 55.5$ \\
\hline Proximal branching density & $8.4 \pm 5.4$ & $8.3 \pm 11.0$ & $7.3 \pm 13.2$ & $6.9 \pm 4.4$ \\
\hline PD cross-sectional area $\left(\mu \mathrm{m}^{2}\right)$ & $27.6 \pm 10.3$ & $11.7 \pm 5.9$ & $18.4 \pm 6.2$ & $20.0 \pm 7.9$ \\
\hline PD maximum Feret diameter $(\mu \mathrm{m})$ & $7.0 \pm 1.2$ & $4.7 \pm 1.2$ & $5.8 \pm 1.1$ & $6.0 \pm 1.2$ \\
\hline PD minimum Feret diameter $(\mu \mathrm{m})$ & $5.1 \pm 1.0$ & $3.2 \pm 1.0$ & $4.2 \pm 0.8$ & $4.5 \pm 1.0$ \\
\hline Vector distance from ONH $(\mu \mathrm{m})$ & $3150 \pm 1241$ & $3076 \pm 1042$ & $3067 \pm 946$ & $3148 \pm 803$ \\
\hline
\end{tabular}

Values are mean $\pm \mathrm{SD}$. 
Table 2. $P C A$ rotated pattern matrix

\begin{tabular}{lccc}
\hline \hline & \multicolumn{3}{c}{ Rotated patter matrix } \\
\cline { 2 - 4 } Variable & Component 1 & Component 2 & Component 3 \\
\hline Soma diameter & $\mathbf{0 . 7 1 3}$ & 0.296 & -0.31 \\
PD maximum Feret & $\mathbf{0 . 9 9 1}$ & -0.047 & -0.009 \\
PD minimum Feret & $\mathbf{0 . 9 7 4}$ & -0.027 & -0.045 \\
PD cross-sectional area & $\mathbf{0 . 9 8 9}$ & -0.024 & 0.007 \\
PBPF area & 0.042 & $\mathbf{0 . 8 5 2}$ & 0.103 \\
SBPF area & 0.097 & $\mathbf{0 . 8 6 4}$ & 0.176 \\
PDN & -0.118 & $\mathbf{0 . 7 1 5}$ & -0.199 \\
PD asymmetry & 0.074 & -0.495 & $\mathbf{0 . 5 2 0}$ \\
PBPF CGO & -0.080 & 0.064 & $\mathbf{0 . 8 7 1}$ \\
SBPF CGO & -0.013 & 0.110 & $\mathbf{0 . 9 2 8}$ \\
& & & \\
\hline \hline
\end{tabular}

$\overline{\text { Bold text denotes component to which each variable gives its greatest }}$ contribution. variance within the data and describes RGC proximal dendritic tree size and symmetry, soma size and dendrite thickness. It correlated positively with soma diameter, primary dendrite crosssectional area, minimum and maximum Feret, SBPF area, and number of PDs. The first discriminant function also showed a negative correlation with PD range. The second discriminant function (which accounted for $22 \%$ of the remaining between group variance) correlated positively with PBPF CGO, describing asymmetry in the proximal dendritic field, with a contribution from soma and dendrite thickness. The third discriminant function, accounting for the remaining $6 \%$ of between group variance; the function showed a negative correlation to SBPF CGO and a positive correlation to PBPF area. The largest determinant of RGC classification is therefore primary dendrite thickness and soma and proximal dendritic tree size, with a smaller contribution of asymmetry of the proximal dendritic tree. This is evident in the clear separation of the group centroids (Fig. 4) where the
A

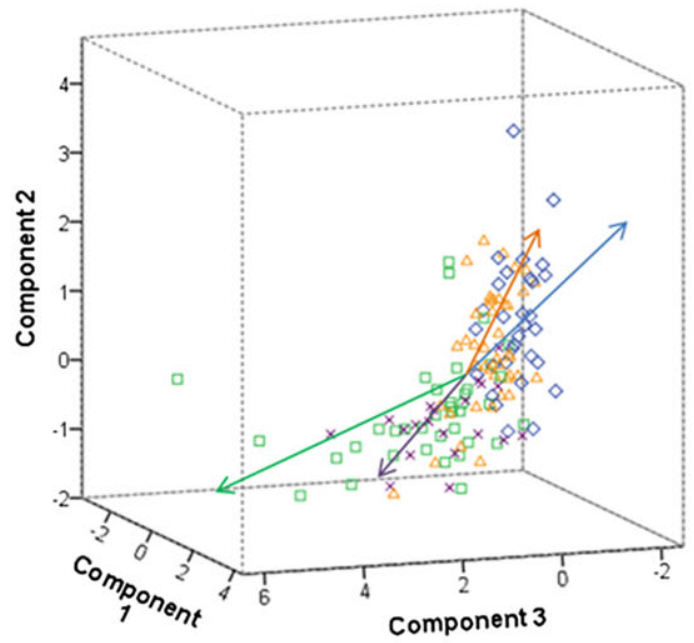

B

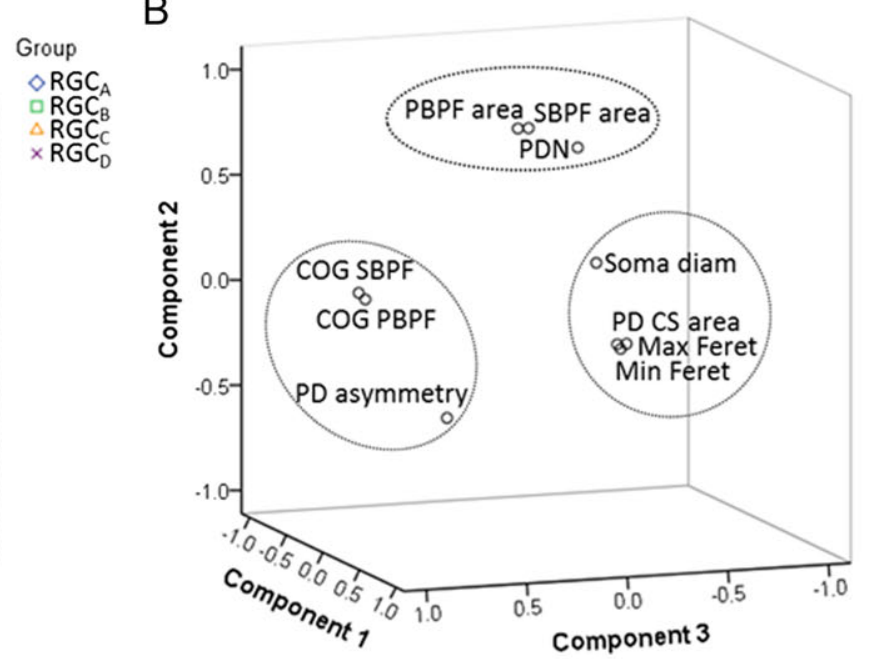

C

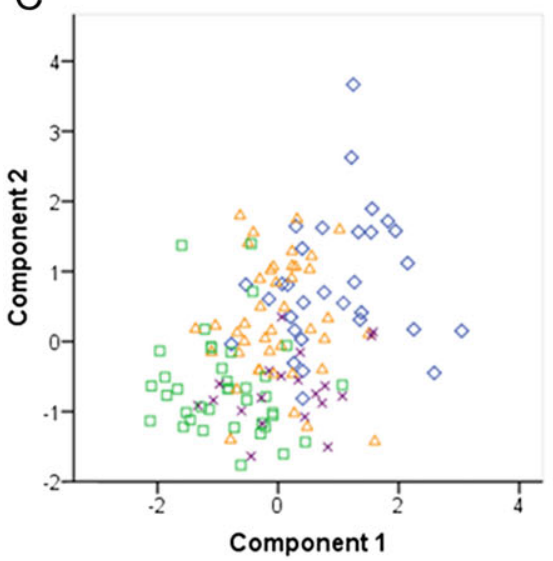

D

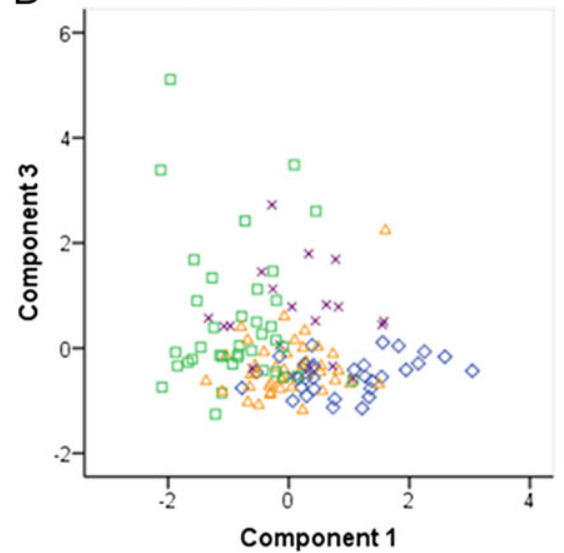

$\mathrm{E}$

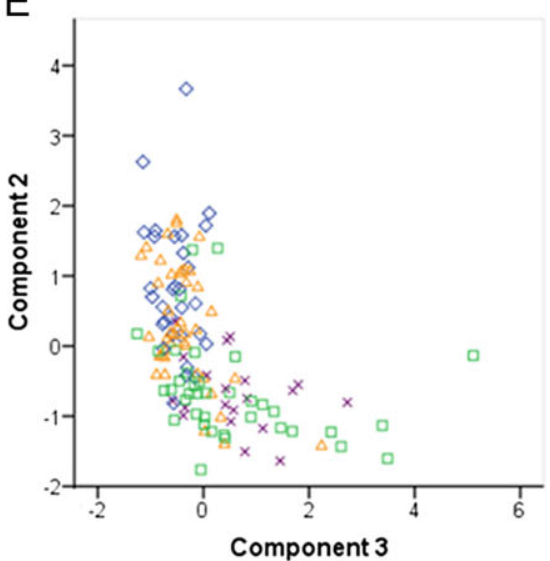

Fig. 5. Principal component analysis of RGCs. PCA generated 3 components to describe variance in RGC morphology. The component scores of each RGC $(n=131)$ for the 3 components are plotted in $X, Y$, and $Z$ dimensions. When labeled according to type $\left(\mathrm{RGC}_{\mathrm{A}}=\mathrm{blue}_{\mathrm{e}}, \mathrm{RGC}_{\mathrm{B}}=\right.$ green, $R_{G C}=$ orange, $R_{C C}=$ purple), this separation of the data corresponds to the $4 \mathrm{RGC}$ types $(\mathbf{A})$. The variable weightings toward each component are plotted in this rotated space $(\mathbf{B})$. The components represent condensed variables derived from correlated or covarying variables (those encompassed by broken lines). Soma diameter, PD cross-sectional area, and minimum and maximum Feret length weight highly on component 1. Primary and SBPF area and PD number weight highly on component 2 while both the PBPF and SBPF center of gravity offset and PD asymmetry weight highly on component 3 . The overlap in RGC size can be seen in the distribution of types along the first and second components $(\mathbf{C})$ however taken together RGC types can be fairly separated based on the soma and proximal dendritic field size where $\mathrm{RGC}_{\mathrm{A}}>$ $R_{G C}>R_{C} C_{D}>R_{B C}$. Inclusion of the third component allows for a separation of RGC types according to asymmetry in the proximal dendritic field as evidenced by clear separation of $\mathrm{RGC}_{\mathrm{B}}$ and $\mathrm{RGC}_{\mathrm{D}}$ from $\mathrm{RGC}_{\mathrm{A}}$ and $\mathrm{RGC}_{\mathrm{C}}$ along the third component $(\mathbf{D}$ and $\mathbf{E})$. 


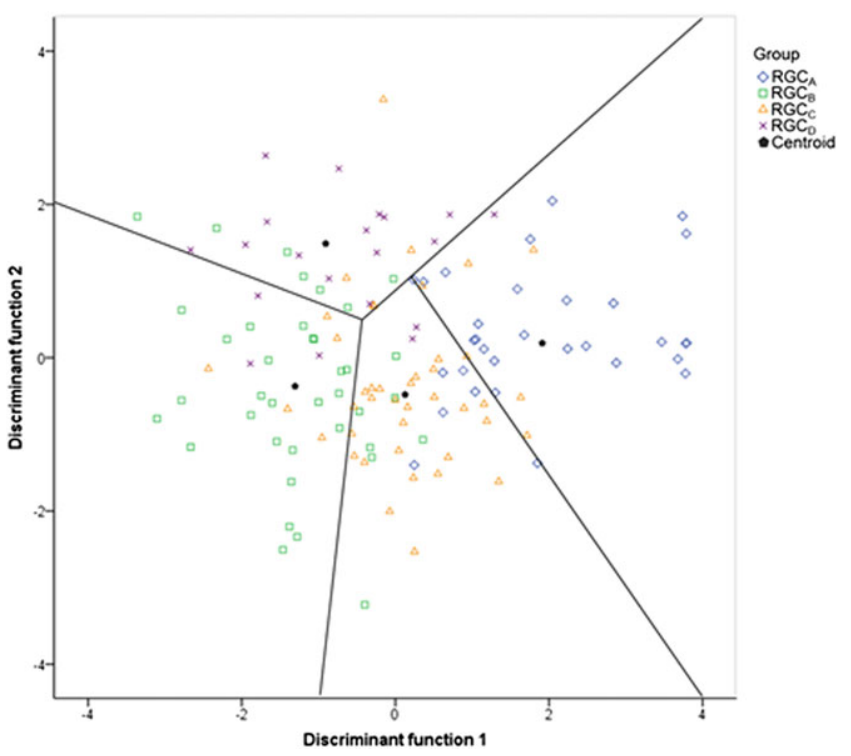

Fig. 6. Discriminant analysis of RGCs. The discriminant scores of each RGC $(n=131)$ are plotted in X, Y dimensions corresponding to the first two discriminant functions. Here variance between groups is maximized so that group centroids (black pentagons) are at maximal distance from each other. RGC classification into a given group is achieved when distance to group centroid is shortest for that given group. The area in which this is true defines the group boundary. When labeled according to type, it can be seen that the majority of RGCs are correctly classified to their group by the discriminant functions.

first discriminant function separates all RGC types in the order of size (where $R_{G C}>R_{A} C_{C}>R_{C} C_{D}>R_{G C}$ ).

\section{RGC types discriminated by proximal dendritic morphology}

The discriminant functions correctly classified $73.3 \%(n=74)$ of RGCs from the training sample and $63.3 \%(n=19)$ from the hold out sample. This was greater than the correct by-chance classification of $25 \%$. Classification is predicted based on RGC group centroids which are summarized in Table 4 . The discriminant scores are plotted in Figure 6 to demonstrate the sub grouping of RGCs. The predicted group percentages for each RGC type are summarized in

Table 3. Correlations between variables and discriminant functions

\begin{tabular}{lccc}
\hline \hline & \multicolumn{3}{c}{ Structure matrix } \\
\cline { 2 - 4 } Variable & Function 1 & Function 2 & Function 3 \\
\hline Soma diameter & $\mathbf{0 . 6 1 9}$ & 0.361 & 0.183 \\
PD maximum Feret & $\mathbf{0 . 5 2 5}$ & 0.378 & 0.107 \\
PD minimum Feret & $\mathbf{0 . 5 4 3}$ & 0.467 & 0.199 \\
PD cross-sectional area & $\mathbf{0 . 5 6 6}$ & 0.416 & -0.028 \\
PBPF area & 0.300 & -0.353 & $\mathbf{0 . 5 6 0}$ \\
SBPF area & $\mathbf{0 . 4 8 7}$ & -0.131 & 0.088 \\
PDN & $\mathbf{0 . 4 8 1}$ & -0.268 & 0.067 \\
PD asymmetry & $\mathbf{- 0 . 5 5 2}$ & 0.483 & -0.233 \\
PBPF CGO & -0.326 & $\mathbf{0 . 4 5 0}$ & -0.302 \\
SBPF CGO & -0.328 & 0.119 & $\mathbf{- 0 . 6 0 5}$ \\
& & &
\end{tabular}

Bold text denotes function to which each variable gives its greatest contribution.
Table 4. $R G C$ group Centroid

\begin{tabular}{lrcr}
\hline \hline & \multicolumn{3}{c}{ Group centroids } \\
\cline { 2 - 4 } Group & Function 1 & Function 2 & Function 3 \\
\hline RGC $_{\mathrm{A}}$ & 1.912 & 0.189 & -0.284 \\
RGC $_{\mathrm{B}}$ & -1.306 & -0.373 & -0.379 \\
RGC $_{\mathrm{C}}$ & 0.126 & -0.048 & 0.427 \\
RGC $_{\mathrm{D}}$ & -0.908 & 1.488 & 0.194 \\
& & & \\
\hline \hline
\end{tabular}

Table 5. RGC groups can usefully be discriminated by primary and secondary dendrite variables. Misclassification followed a similar trend with roughly $25 \%$ misclassified to the RGC type closest in morphology to the correct group. All misclassified RGCs had at least 1 variable (average 3 variables) that was $>1$ standard deviation from the mean of its correct type.

The relationship between population wide variance and between group variance was explored using the component scores generated through PCA as variables to describe the morphology of each RGC. The components alone achieved only a correct classification in $62.6 \%$ of the training sample $(n=101)$ and $60 \%$ of the hold out sample $(n=30)$. This indicates that population variance in morphology does not adequately describe the difference between groups.

\section{$R G C$ subgroups cannot be differentiated}

Discriminant analysis was performed as described initially but where RGCs were grouped according to the subgroups described by Sun et al. (2002a). The original hold out sample did not contain two of the subgroups and so the sample size was increased to 40 RGCs following the same random assignment method. Again, prior probability was calculated based on subgroup frequency. Correct classification was achieved in 53.8\% $(n=49)$ of RGCs from the training sample and 35\% $(n=14)$ from the hold out sample. While greater than the correct by chance classification (8.3\%), it does not yield adequate classification indicating that the ability to resolve subgroups is beyond this model.

\section{Discussion}

Our data support the concept that RGCs in the rat retina can be classified using dendrite features that are relatively resistant to early degenerative events. It is important to note that the classification is not as precise as that provided by the dendritic tree as a whole (which includes tertiary and higher dendritic structures); however, the model provides a framework to check for labeling bias in the analysis of RGCs in retinal disease. Our data indicate that eccentricity is not a factor that biased RGC classification on the basis of soma size.

We show that RGCs can be discriminated on the basis of soma and proximal dendritic field size. This is in agreement with Sun et al. (2002a) and other classifications based on RGC morphology where larger $\mathrm{RGC}_{\mathrm{A}}$ and $\mathrm{RGC}_{\mathrm{C}}$ are separated from the smaller $\mathrm{RGC}_{\mathrm{B}}$ and $\mathrm{RGC}_{\mathrm{D}}$ based on soma and dendritic field size. We also demonstrate dendrite thickness as an effective parameter for classifying RGC types due to its strong relationship with other features of dendritic tree morphology. Primary dendrite thickness has been found to correlate with soma size, dendritic field size, total dendritic 
Table 5. Summary of classification

\begin{tabular}{|c|c|c|c|c|c|c|c|}
\hline & \multirow[b]{2}{*}{ A priori RGC type } & \multicolumn{4}{|c|}{ Predicted RGC type } & \multirow[b]{2}{*}{ Number of a priori RGC } & \multirow{2}{*}{$\begin{array}{c}\text { Percentage correctly } \\
\text { classified }\end{array}$} \\
\hline & & $\mathrm{RGC}_{\mathrm{A}}$ & $\mathrm{RGC}_{\mathrm{B}}$ & $\mathrm{RGC}_{\mathrm{C}}$ & $\mathrm{RGC}_{\mathrm{D}}$ & & \\
\hline \multirow[t]{4}{*}{ Training sample $(n=101)$} & $\mathrm{RGC}_{\mathrm{A}}$ & 18 & 0 & 6 & 0 & 24 & $75 \%$ \\
\hline & $\mathrm{RGC}_{\mathrm{B}}$ & 0 & 18 & 7 & 3 & 28 & $64 \%$ \\
\hline & $\mathrm{RGC}_{\mathrm{C}}$ & 2 & 4 & 26 & 2 & 34 & $77 \%$ \\
\hline & $\mathrm{RGC}_{\mathrm{D}}$ & 1 & 2 & 0 & 12 & 15 & $80 \%$ \\
\hline \multirow[t]{4}{*}{ Hold-out sample $(n=30)$} & $\mathrm{RGC}_{\mathrm{A}}$ & 5 & 0 & 2 & 0 & 7 & $71 \%$ \\
\hline & $\mathrm{RGC}_{\mathrm{B}}$ & 0 & 7 & 2 & 1 & 10 & $70 \%$ \\
\hline & $\mathrm{RGC}_{\mathrm{C}}$ & 2 & 0 & 5 & 1 & 8 & $63 \%$ \\
\hline & $\mathrm{RGC}_{\mathrm{D}}$ & 0 & 0 & 3 & 2 & 5 & $40 \%$ \\
\hline
\end{tabular}

length, and branching density in rat triceps surae motoneurons (Chen \& Wolpaw, 1994) and cat spinal $\alpha$-and $\gamma$-motoneurons (Ulfhake \& Cullheim, 1981; Ulfhake \& Kellerth, 1981), all parameters used in the classification of RGC types. We also show that asymmetry in the proximal dendritic tree has a small discriminatory effect on RGC types.

The use of DiOlistic labeling resulted in a number of cells being excluded from analysis as the soma and proximal dendrites were obscured by excessive labeling. However, we justify the use of DiOlistic (rather than Biolistic) labeling since this is not contingent on cell health (Honig \& Hume, 1986). Studies in the mouse glaucoma model suggest that the level of reporter expression may be affected by neuronal viability (Williams et al., 2013). This is vital given that the classification purpose is for use in degeneration where Biolistics is affected by the viability of transcriptional pathways necessary for the expression of fluorescent markers. Ballistic labeling techniques are open to the criticism that they bias for larger cell types since the likelihood of a hit increases with cell size. We did not observe this bias in our sample suggesting that the difference in cell soma size is insufficient to have a large effect on labeling probability. A higher proportion of $\mathrm{RGC}_{\mathrm{A}}$ (at $24 \%$ ) was found in comparison to the $18 \%$ reported by Sun et al. (2002a); however, this was much smaller than the $60 \%$ previously reported (Huxlin \& Goodchild, 1997). If soma size were the largest determinant of a given cell being labeled, one would expect a higher proportion of $\mathrm{RGC}_{\mathrm{A}}$ than $\mathrm{RGC}_{\mathrm{B}}$; almost twice as many $\mathrm{RGC}_{\mathrm{B}}$ to $\mathrm{RGC}_{\mathrm{A}}$ were labeled in this study. In fact, retrograde labeling methods, such as those used by Huxlin and Goodchild (1997) bias toward the labeling of larger cell types, which further justifies the use of DiOlistics.

The robust discrimination between cell types is important in the study of degeneration, both in terms of reducing pseudodegenerative effects arising from intertype differences in dendritic morphology and also in investigating intertype susceptibility to disease. Type specific degeneration has been noted in the primate (Weber et al., 1998), cat (Shou et al., 2003), mouse (Leung et al., 2011; Feng et al., 2013), and rat (Thanos, 1988) but not in DBA/2J (Jakobs et al., 2005) or thy1-YFP-H transgenic mice with experimental glaucoma (Kalesnykas et al., 2012). The Sholl plots in Fig. 3 demonstrate the differences in branching pattern and density between RGC types and illustrate effectively how disparity in cell types between populations could be confused for atrophy in studies of degeneration.

We anticipate that the chosen parameters will remain stable during degeneration given that significant reduction in branching occurs only in the distal region of the dendritic tree, as illustrated in
Fig. 1. Measurement of soma diameter is also included based on its greater resistance to degeneration than the dendritic tree. The dendritic tree exhibits the earliest change in RGCs under degeneration while the soma only undergoes substantial change with advanced/ chronic glaucoma (Weber et al., 1998). Significant change in RGC soma size in the rat is seen in chronic glaucoma models, however is similar across all retinal regions (Urcola et al., 2006) and so is unlikely to vary between types. The ability of the system to classify cell types may therefore diminish with substantial degeneration, as soma and secondary and primary dendrite degeneration occurs. The inclusion of dendritic stratification depth within the IPL could enhance the correct classification of RGC types, in particular the classification of $\mathrm{RGC}_{\mathrm{D}}$ due to their bistratified dendritic tree.

The reproducibility of this model requires testing on a range of degenerated RGC populations, the scope of which was beyond the current study. However, applying the model to a degenerated population of RGCs would not serve to validate the model given that the correct type of an RGC cannot be determined from its postdegenerative morphology while following the degeneration of single RGCs over time is reliant on reporters whose expression is compromised in degeneration.

We would not anticipate complete neuronal classification in view of the large variability in RGC morphology and overlap between groups for any single morphological criterion. Consistent with this, all misclassified RGCs exhibited one or more variable measurement $>1 \mathrm{SD}$ from the mean of its type. The correct subtype classification of just over a third of RGCs provides encouragement for success if given a larger population. It is also possible that the misclassified atypical cells represent previously unidentified cell types given that the number of RGC types currently defined in the closely related mouse is 22 (Voelgyi et al., 2009). The main role of this classification procedure is therefore not the definitive classification of all RGC types but to provide a degree of confidence regarding RGC proportions in a sample population where traditional methods of classification are vulnerable to bias.

\section{Acknowledgments}

The authors gratefully acknowledge Dr. Jonathan Gillard for his advice on the statistical methodology for classification.

\section{Funding}

Biotechnology and Biological Research Council grant number BB/ F016352/1 and Cardiff University. 


\section{References}

Barber, A.J., Lieth, E., Khin, S.A., Antonetti, D.A., Buchanan, A.G. \& GARDNER, T.W. (1998). Neural apoptosis in the retina during experimental and human diabetes - Early onset and effect of insulin. Journal of Clinical Investigation 102, 783-791.

Boycott, B.B. \& Wassle, H. (1974). Morphological types of ganglioncells of domestic cats retina. Journal of Physiology-London 240, 397.

Brown, S.P., HE, S.G. \& Masland, R.H. (2000). Receptive field microstructure and dendritic geometry of retinal ganglion cells. Neuron 27, 371-383.

Chen, X.Y. \& Wolpaw, J.R. (1994). Triceps surae motoneuron morphology in the rat - A quantitative light-microscopic study. Journal of Comparative Neurology 343, 143-157.

Citron, M.C., Emerson, R.C. \& Levick, W.R. (1988). Nonlinear measurement and classification of receptive-fields in cat retinal ganglion-cells. Annals of Biomedical Engineering 16, 65-77.

Coelho, R.C., Di Gesu, V., Lo Bosco, G., Tanaka, J.S. \& Valenti, C. (2002). Shape-based features for cat ganglion retinal cells classification. Real-Time Imaging 8, 213-226.

Coombs, J., van der List, D., Wang, G.Y. \& Chalupa, L.M. (2006). Morphological properties of mouse retinal ganglion cells. Neuroscience 140, 123-136.

DACEY, D.M. (1993). Morphology of a small-field bistratified ganglioncell type in the macaque and human retina. Visual Neuroscience 10, 1081-1098.

Enroth-Cugell, C. \& Robson, J.G. (1966). The contrast sensitivity of retinal ganglion cells of the cat. The Journal of Physiology 187, $517-552$.

Feng, L., Zhao, Y., Yoshida, M., Chen, H., Yang, J.F., Kim, T.S., CANG, J.H., Troy, J.B. \& LiU, X.R. (2013). Sustained ocular hypertension induces dendritic degeneration of mouse retinal ganglion cells that depends on cell type and location. Investigative Ophthalmology \& Visual Science 54, 1106-1117.

Gan, W.B., Grutzendler, J., Wong, W.T., Wong, R.O.L. \& Lichtman, J.W. (2000). Multicolor "DiOlistic" labeling of the nervous system using lipophilic dye combinations. Neuron 27, 219-225.

Gutierrez, H. \& DaVIES, A.M. (2007). A fast and accurate procedure for deriving the Sholl profile in quantitative studies of neuronal morphology. Journal of Neuroscience Methods 163, 24-30.

HochSTEIN, S. \& SHAPLEY, R.M. (1976). Quantitative-analysis of retinal ganglion-cell classifications. Journal of Physiology-London 262, 237-264

HonIG, M.G. \& Hume, R.I. (1986). Fluorescent carbocyanine dyes allow living neurons of identified origin to be studied in long-term cultures Journal of Cell Biology 103, 171-187.

HuxLIN, K.R. \& GoodCHILD, A.K. (1997). Retinal ganglion cells in the albino rat: Revised morphological classification. Journal of Comparative Neurology 385, 309-323.

Jakobs, T.C., Libby, R.T., Ben, Y.X., John, S.W.M. \& Masland, R.H. (2005). Retinal ganglion cell degeneration is topological but not cell type specific in DBA/2J mice. Journal of Cell Biology 171, 313-325.

Kalesnykas, G., Oglesbi, E.N., Zack, D.J., Cone, F.E., Steinhart, M.R., Tian, J., Pease, M.E. \& Quigley, H.A. (2012). Retinal ganglion cell morphology after optic nerve crush and experimental glaucoma. Investigative ophthalmology \& visual science 53, 3847-3857.

Leung, C.K., Weinreb, R.N., Li, Z.W., Liu, S., Lindsey, J.D., Choi, N., Liu, L., Cheung, C.Y., Ye, C., Qiu, K., Chen, L.J., Yung, W.H., Crowston, J.G., Pu, M., So, K.F., PAnG, C.P. \& LaM, D.S.C. (2011). Long-term in vivo imaging and measurement of dendritic shrinkage of retinal ganglion cells. Investigative Ophthalmology \& Visual Science 52, 1539-1547.

Longair, M.K., BaKer, D.A. \& Armstrong, J.D. (2011). Simple neurite tracer: open source software for reconstruction, visualisation, and analysis of neuronal processes. Bioinformatics 27, 2453-2454.

Morgan, J.E., Datta, A.V., Erichsen, J.T., Albon, J. \& Boulton, M.E (2006). Retinal ganglion cell remodelling in experimental glaucoma. In Retinal Degenerative Diseases, ed. Hollyfield, J.G., ANDERSON, R.E. \& LAVAIL, M.M., pp. 397-402.

O'Brien, B.J., Isayama, T., Richardson, R. \& Berson, D.M. (2002). Intrinsic physiological properties of cat retinal ganglion cells. Journal of Physiology-London 538, 787-802.

PeICHL, L. (1991). Alpha ganglion-cells in mammalian retinae - Common properties, species-differences, and some comments on other ganglioncells. Visual Neuroscience 7, 155-169.

Qu, J., Wang, D. \& Grosskreutz, C.L. (2010). Mechanisms of retinal ganglion cell injury and defense in glaucoma. Experimental Eye Research 91, 48-53.

Schindelin, J., Arganda-Carreras, I., Frise, E., Kaying, V., Longair, M. \& Pietzsch, T. (2012). Fiji: an open-source platform for biologicalimage analysis. Nature Methods 9, 676-682.

Shou, T.D., Liu, J., Wang, W., Zhou, Y.F. \& Zhao, K.X. (2003). Differential dendritic shrinkage of alpha and beta retinal ganglion cells in cats with chronic glaucoma. Investigative Ophthalmology \& Visual Science 44, 3005-3010.

Sun, W.Z., LI, N. \& He, S.G. (2002a). Large-scale morphological survey of rat retinal ganglion cells. Visual Neuroscience 19, 483-493.

Sun, W.Z., Li, N. \& He, S.G. (2002b). Large-scale morphological survey of mouse retinal ganglion cells. Journal of Comparative Neurology 451 $115-126$.

Thanos, S. (1988). Alterations in the morphology of ganglion-cell dendrites in the adult-rat retina after optic-nerve transection and grafting of peripheral-nerve segments. Cell and Tissue Research 254, 599-609.

Ulfhake, B. \& Cullheim, S. (1981). A quantitative light microscopic study of the dendrites of cat spinal gamma-motoneurons after intracellular staining with horseradish-peroxidase. Journal of Comparative Neurology 202, 585-596.

Ulfhake, B. \& Kellerth, J.O. (1981). A quantitative light microscopic study of the dendrites of cat spinal alpha-motoneurons after intracellular staining with horseradish-peroxidase. Journal of Comparative Neurology 202, 571-583.

Urcola, J.H., Hernandez, M. \& Vecino, E. (2006). Three experimental glaucoma models in rats: Comparison of the effects of intraocular pressure elevation on retinal ganglion cell size and death. Experimental Eye Research 83, 429-437.

Voelgyi, B., Chheda, S. \& Bloomfield, S.A. (2009). Tracer coupling patterns of the ganglion cell subtypes in the mouse retina. Journal of Comparative Neurology 512, 664-687.

Votruba, M., Moore, A.T. \& Bhattacharya, S.S. (1998). Clinical features, molecular genetics, and pathophysiology of dominant optic atrophy. Journal of Medical Genetics 35, 793-800.

Weber, A.J., Kaufman, P.L. \& Hubbard, W.C. (1998). Morphology of single ganglion cells in the glaucomatous primate retina. Investigative Ophthalmology \& Visual Science 39, 2304-2320.

Williams, P.A., Howell, G.R., Barbay, J.M., Braine, C.E., Sousa, G.L., JoHn, S.W.M. \& Morgan, J.E. (2013). Retinal ganglion cell dendritic atrophy in DBA/2J glaucoma. PLOS One 8, e72282.

Williams, P.A., Morgan, J.E. \& Votruba, M. (2010). Opa1 deficiency in a mouse model of dominant optic atrophy leads to retinal ganglion cell dendropathy. Brain 133, 2942-2951. 